\title{
A Clinical Scoring System to Predict the Development of Bronchopulmonary Dysplasia
}

\author{
Tugba Gursoy, MD ${ }^{1}$ Mutlu Hayran, MD ${ }^{2}$ Hatice Derin, MD ${ }^{3}$ Fahri Ovali, MD
}

${ }^{1}$ Department of Neonatology, School of Medicine, KOC University, Istanbul, Turkey

2 Department of Preventive Oncology, Faculty of Medicine, Hacettepe

Address for correspondence Tugba Gursoy, MD, American Hospital, NICU, Guzelbahce sok. No. 20, 34365. Nisantasi, Sisli, Istanbul, Turkey University, Ankara, Turkey

${ }^{3}$ Department of Pediatrics, Zeynep Kamil Maternity and Children's Research and Training Hospital, Istanbul, Turkey

${ }^{4}$ Department of Neonatology, Zeynep Kamil Maternity and Children's Research and Training Hospital, Istanbul, Turkey

\begin{abstract}
Keywords

- bronchopulmonary dysplasia

- prediction

- risk factors

- prematurity

Objective This study aims to develop a scoring system for the prediction of bronchopulmonary dysplasia (BPD).

Methods Medical records of 652 infants whose gestational age and birth weight were below 32 weeks and $1,500 \mathrm{~g}$, respectively, and who survived beyond 28th postnatal day were reviewed retrospectively. Logistic regression methods were used to determine the clinical and demographic risk factors within the first 72 hours of life associated with BPD, as well as the weights of these factors on developing BPD. Predictive accuracy of the scoring system was tested prospectively at the same unit.

Results Birth weight, gestational age, gender, presence of respiratory distress syndrome, patent ductus arteriosus, intraventricular hemorrhage, hypotension were the most important risk factors for BPD. Therefore, a scoring system (BPD-TM score) ranging from 0 to 13 and grouped in four tiers (0-3: low, 4-6: low intermediate, 7-9: high intermediate, and 10-13: high risk) was developed based on these factors. Below the score of $4,4.1 \%$ of infants (18/436), above the score of $9,100 \%(29 / 29)$ of the infants developed BPD. The score was validated successfully in 172 infants.

Conclusion With this easy to use scoring system, one can predict the neonate at risk for BPD at 72 hours of life and direct preventive measures toward these infants.
\end{abstract}

\section{Clinical Perspective}

This is a simple and easy to use clinical scoring system that predicts the development of bronchopulmonary dysplasia (BPD) in very low-birth-weight infants. With this system, one can predict the neonate at 72 hours of life at risk for BPD. One can direct preventive measures to these vulnerable neonates before any irreversible damage takes place.

BPD is the most common serious pulmonary morbidity in premature infants. ${ }^{1}$ As more premature infants survive, its

received

June 17, 2014

accepted after revision

August 12, 2014

published online

October 6, 2014 incidence increases, especially among extremely low-birthweight infants, despite the recent advances in neonatology. ${ }^{2}$

There is no specific treatment for BPD once it is established. ${ }^{3}$ Care during the 1 st postnatal week is vital as intervention in the dynamic process of developing lung tissue during this period allows prevention of damage and abnormal healing. ${ }^{4}$ Early prediction of BPD facilitates the institution of treatment or preventive measures before irreparable damage takes place and also permits appropriate enrollment of cases in clinical trials.
Copyright $\odot 2015$ by Thieme Medical Publishers, Inc., 333 Seventh Avenue, New York, NY 10001, USA. Tel: +1(212) 584-4662.
DOI http://dx.doi.org/ 10.1055/s-0034-1393935. ISSN 0735-1631. 
Several studies have tried different approaches to identify an accurate method for prediction of BPD. ${ }^{2-12}$ Ventilator parameters, birth weight, gestational age, clinical variables, ${ }^{4,5}$ pulmonary artery pressure, ${ }^{7}$ pulmonary compliance, ${ }^{8,9}$ total pulmonary resistance, ${ }^{8,9}$ pulmonary impedance, ${ }^{9}$ radiological findings, $4,10,11$ and cytological examination of the bronchoalveolar fluid ${ }^{10}$ were used to predict BPD. In this study, we aimed to develop a simple and easy to use the clinical scoring system to predict the development of BPD in very low-birthweight infants.

\section{Methods}

Medical records of 652 infants who had gestational age and birth weight $\leq 32$ weeks and $\leq 1,500 \mathrm{~g}$, respectively, at birth and survived beyond 28th postnatal day were reviewed retrospectively (derivation cohort). Infants with congenital anomalies were excluded. The babies included in the study were born at Zeynep Kamil Maternity and Children's Training and Research Hospital between the years 2006 and 2009.

Antenatal, natal, and postnatal features together with the laboratory findings of the infants were recorded.

An infant was considered to receive antenatal steroids when if the mothers received a complete course of steroids (12 $\mathrm{mg} /$ dose intramuscular betamethasone, given at 12 or 24 hours interval). Chorioamnionitis was diagnosed with the presence of typical clinical findings such as fever, uterine fundal tenderness, maternal tachycardia ( $>100 / \mathrm{min})$, fetal tachycardia ( $>160 / \mathrm{min}$ ) and purulent or foul amniotic fluid. ${ }^{13}$ Cases with birth weight, lower than 10th percentile for gestational age, according to the Turkish growth charts, were classified as intrauterine growth retarded. ${ }^{14}$ Neonates who needed positive pressure ventilation in the delivery room were accepted as having delivery room resuscitation. Perinatal asphyxia was considered in infants with cord $\mathrm{pH}<7.1$, or an arterial $\mathrm{pH}<7.1$ or base deficit of 12 or more within 60 minutes of birth. ${ }^{15}$ Fetal distress was diagnosed in pregnancies where the fetal biophysical profile was less than three. ${ }^{16}$ Hypothermia was diagnosed in infants whose body temperature was below $36^{\circ} \mathrm{C}$ measured from the axilla. An infant was accepted as thrombocytopenic, if platelet count was less than $100,000 / \mathrm{mm}^{3}$, neutropenic if neutrophil count was less than $1,000 / \mathrm{mm}^{3}$, anemic if hemoglobin level was less than $120 \mathrm{~g} / \mathrm{L}$, hypoglycemic if serum glucose level was less than $2.6 \mathrm{mmol} / \mathrm{L}$ and hypocalcemic if serum calcium levels were less than $2 \mathrm{mmol} / \mathrm{L}$. Respiratory distress syndrome (RDS) was defined as respiratory distress with characteristic radiographic findings. ${ }^{17}$ Hypotension was defined in infants whose mean blood pressure was lower than the gestational age and needed inotrope treatment. ${ }^{18}$ Intraventricular hemorrhage (IVH) was classified according to the Papile classification. ${ }^{19}$ Only culture positive early onset sepsis was denoted as sepsis. Echocardiography was performed to all infants. Hemodynamically significant patent ductus arteriosus (PDA) was defined when; (1) ductal diameter was measured more than $1.6 \mathrm{~mm}$, (2) the PDA flow was growing or pulsatile with left-to-right shunt, (3) there was reversal of flow in the descending aorta or (4) reversal or absence of blood flow in organ vessels. ${ }^{20}$ Infants who were on oxygen for at least 28 days were defined as BPD. These infants were reevaluated at 36 weeks of postmenstrual age and if the baby was breathing room air, BPD was classified as mild, if the need for oxygen was less than $30 \%$, it was called moderate BPD, and if the need was more than $30 \%$ oxygen and/or there was also a need for positive pressure ventilation (PPV) or nasal continuous positive airway pressure (nCPAP), then the BPD was severe. ${ }^{21}$

Statistical analyses were performed using the SPSS software. The univariate analyses used to identify variables associated with BPD (present/absent) were chi-square, Fisher exact, Student's $t-$, and Mann-Whitney U tests, where appropriate. For the multivariate analysis, factors identified to be possibly associated with BPD in univariate analyses (with $p$-values $<0.25)$ were further entered into the logistic regression model to determine independent predictors of patient outcome. Hosmer-Lemeshow goodness of fit statistics and correlation matrices of parameter estimates were investigated to assess model fit. Spearman correlation coefficients were used to assess the correlation between the BPD severity and the devised score.

An arbitrary scoring system (BPD-TM score, a proposed test measure for BPD) using the findings at day 3 was developed by assigning points to categories significantly associated with BPD risk, taking into account their risk ratios (RR) from the logistic regression model. After determination of the optimal clinical scoring system, it was validated prospectively on all neonatal intensive care unit (NICU) admissions $\leq 32$ weeks of gestation and $\leq 1,500 \mathrm{~g}$ birth weight at the same hospital between January 2012 and December 2012 (validation cohort), and the predictive accuracy of the scoring system was tested. A flow diagram showing the infants admitted to NICU in 2012 is given in - Fig. 1.

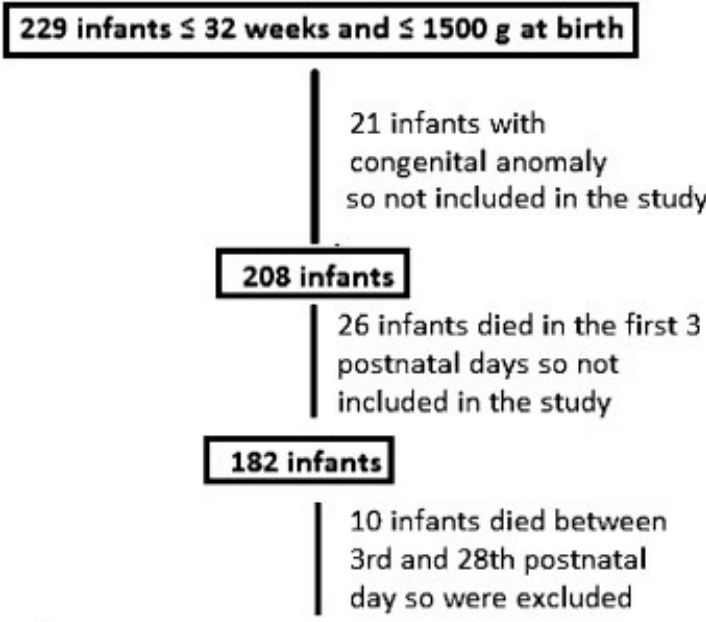

172 infants were included in the study

Fig. 1 Flow diagram showing the infants admitted to NICU in 2012 (validation cohort). In our unit only inborn infants are hospitalized. In 2012 a total of 10,049 infants were born in our hospital and 1,397 infants were admitted to NICU where 943 were premature. NICU, neonatal intensive care unit. 
Table 1 Antenatal, natal, and postnatal features together with laboratory findings of the infants for the derivation cohort (mean \pm standard deviations or counts with percentages are provided)

\begin{tabular}{|c|c|c|c|}
\hline Features & $\begin{array}{l}\text { BPD (-) } \\
n=502\end{array}$ & $\begin{array}{l}\text { BPD (+) } \\
n=150\end{array}$ & $p$-Value \\
\hline \multicolumn{4}{|l|}{ Antenatal features } \\
\hline Mother's age $(y)$ & $27.4 \pm 6$ & $28.3 \pm 6.5$ & 0.12 \\
\hline Preeclampsia, $n(\%)$ & 90 (17.9) & $18(12)$ & 0.08 \\
\hline Diabetes, $n(\%)$ & $8(1.6)$ & $2(1.3)$ & 1 \\
\hline Chorioamnionitis, $n(\%)$ & $15(3)$ & $10(6.7)$ & 0.04 \\
\hline PPROM, $n(\%)$ & $127(25.3)$ & $35(23.3)$ & 0.62 \\
\hline Oligohydramnios, $n(\%)$ & $63(12.5)$ & $19(12.7)$ & 0.98 \\
\hline Multiple pregnancies, $n(\%)$ & $128(25.5)$ & $34(23)$ & 0.78 \\
\hline Steroid administration, $n(\%)$ & $359(71.5)$ & $102(68)$ & 0.17 \\
\hline IUGR, $n(\%)$ & $43(8.6)$ & $12(8)$ & 0.49 \\
\hline Fetal distress, $n(\%)$ & $73(14.5)$ & $23(15.3)$ & 0.82 \\
\hline \multicolumn{4}{|l|}{ Natal features } \\
\hline Delivery route (CS), $n(\%)$ & $383(76.3)$ & $98(65.3)$ & 0.007 \\
\hline Apgar score & $7.8 \pm 1.1$ & $7.1 \pm 1.2$ & $<0.001$ \\
\hline Perinatal asphyxia, $n(\%)$ & $13(2.6)$ & $10(6.7)$ & 0.018 \\
\hline Delivery room resuscitation, $n(\%)$ & $214(42.6)$ & $110(73.3)$ & $<0.001$ \\
\hline Gender (male), $n$ (\%) & $231(46)$ & $88(58.7)$ & 0.006 \\
\hline Gestational age (wk) & $29.9 \pm 1.6$ & $27.8 \pm 2.1$ & $<0.001$ \\
\hline Birth weight $(\mathrm{g})$ & $1,280 \pm 181$ & $1,009 \pm 211$ & $<0.001$ \\
\hline \multicolumn{4}{|l|}{ Postnatal features } \\
\hline Intubation, $n(\%)$ & 95 (18.9) & $134(89.3)$ & $<0.001$ \\
\hline RDS, $n(\%)$ & $78(15.5)$ & $111(74)$ & $<0.001$ \\
\hline Hypothermia, $n$ (\%) & $8(1.6)$ & $28(18.7)$ & $<0.001$ \\
\hline Hypotension, $n(\%)$ & $13(2.6)$ & $49(32.3)$ & $<0.001$ \\
\hline PDA, $n(\%)$ & $33(6.6)$ & $82(54.7)$ & $<0.001$ \\
\hline IVH/GMH (all grades), $n$ (\%) & $24(4.8)$ & $45(30)$ & $<0.001$ \\
\hline Sepsis, $n(\%)$ & $30(6)$ & $18(12)$ & 0.013 \\
\hline Pulmonary hemorrhage, $n(\%)$ & $5(1)$ & $18(12)$ & $<0.001$ \\
\hline Maximum amount of fluid administered during the first $3 \mathrm{~d}(\mathrm{~mL} / \mathrm{kg} / \mathrm{d})$ & $111 \pm 15$ & $113.3 \pm 20.4$ & 0.12 \\
\hline \multicolumn{4}{|l|}{ Laboratory findings } \\
\hline Neutropenia, $n(\%)$ & $32(6.4)$ & $16(10.7)$ & 0.08 \\
\hline Thrombocytopenia, $n(\%)$ & $118(23.5)$ & $54(36)$ & 0.002 \\
\hline Anemia, $n(\%)$ & $37(7.4)$ & $20(13.3)$ & 0.023 \\
\hline Hypoglycemia, $n(\%)$ & $63(12.5)$ & $18(12)$ & 0.86 \\
\hline Hypocalcemia, $n(\%)$ & $76(15.1)$ & $53(35.3)$ & $<0.001$ \\
\hline
\end{tabular}

Abbreviations: CS, cesarean section; IUGR, intrauterine growth deficiency; IVH/GMH, intraventricular hemorrhage/germinal matrix hemorrhage; PDA, patent ductus arteriosus; PPROM, preterm premature rupture of membranes; RDS, respiratory distress syndrome.

The study was approved by the local ethics committee and informed consent was obtained from the parents.

\section{Results}

Of the 652 infants included in the study (derivation cohort), 150 (23\%) developed BPD. A total of 99 (66\%) infants had mild
BPD, 32 (21.3\%) infants had moderate, and 19 (12.7\%) infants had severe BPD. Antenatal features of the infants who developed BPD were not different from those who did not develop BPD except for chorioamnionitis that has a higher incidence in infants with BPD (-Table 1). The Cesarean section ratio was higher in infants without BPD ( - Table 1). Apgar scores, birth weight, gestational age of the infants without BPD were 
higher than the ones with BPD. Infants with BPD were resuscitated in the delivery room more than the ones without BPD and the incidence of perinatal asphyxia was higher in these infants. Males predominated in the BPD group (-Table 1). Incidence of intubation, RDS, hypothermia, hypotension, PDA, IVH, sepsis, pulmonary hemorrhage were all higher in the infants with BPD. However, maximum amount of fluid administered during the first 3 days of life was not different between the infants with and without BPD (-Table 1).

None of the babies received prophylactic surfactant treatment. All the infants were put on nasal CPAP first and they were intubated if they did not tolerate nasal ventilation. Infants with RDS received surfactant as early rescue therapy. None of the patients received steroid treatment for the prophylaxis of BPD.

Thrombocytopenia, anemia, and hypocalcemia in the 1st day of life were significantly higher in infants who subsequently developed BPD (-Table 1 ).

BPD-TM score was developed by assigning points to categories significantly associated with BPD risk, taking into account the RR from the logistic regression model. Points of $0,1,2$, and 3 were assigned to risk categories with RR of 1,2 to 3.5, 3.5 to 6.5 , and $6.5+$, respectively (-Table 2 ). For practical clinical use - Table 3 is also presented as a simpler version of - Table 2. The final BPD-TM score ranged from 0 to 13 . The receiver operator characteristics (ROC) curve analysis was used to investigate the predictive characteristics of the BPD-TM score. The score was further grouped into four risk categories of low $(<4)$, low intermediate (4-6), high intermediate (7-9), and high ( $>9$ ), optimizing the predictive values of lowest and highest categories. If the BPD-TM score was less than 4 , only $4.1 \%$ of infants (18/436) developed BPD. When the BPD-TM score was between 4 and 6 , the percentage of infants developing BPD was 39.8\% (47/118) and between 7 and 9 , it was $81.2 \%$ (56/69). Above the score of 9, 100\% (29 out of 29) of the infants developed BPD.

The BPD-TM scores were further used prospectively in 172 neonates (validation cohort). Comparison of these cohorts is shown in - Table 4. The ROC curves plotted for both the derivation and validation cohort presented in - Fig. $\mathbf{2}$ yield area under the curve values of 0.930 (standard error [SE]:0.012) and 0.903 (SE:0.022), respectively.

The predictive ability of the BPD-TM score for each category in the derivation, validation, and entire cohort was calculated. The negative predictive values of a score less than 4 were 95.9, 98.6, and 96.2\%, while positive predictive values of a score more than 9 were $100,89.5$, and $95.8 \%$ in the derivation, validation, and the combined datasets, respectively. Moreover the severity of BPD (mild, moderate, and severe) highly correlated with BPD-TM score in all datasets (derivation dataset; $r=0.65, p<0.001$, validation dataset; $r=0.64$, $p<0.001$, entire dataset; $\mathrm{r}=0.64, p<0.001$ ) (-Fig. 3 ). As the severity of BPD increases, median score values increased in each dataset.

\section{Discussion}

Clinicians and researchers may need to quantify the morbidity risk for a premature infant for many different reasons. As parents are likely to be advised about their child's BPD risk by the clinician, the BPD score may inform the clinician well and

Table 2 BPD-TM scoring system and associated risks of BPD with each category

\begin{tabular}{|l|l|l|l|}
\hline Factor & Value & $\begin{array}{l}\text { Risk ratio }^{\text {a }} \\
(\mathbf{9 5 \%} \text { Cl) }\end{array}$ & Points \\
\hline \multirow{4}{*}{ Birth weight (g) } & $>1,200$ & 1 (ref. cat.) & 0 \\
\cline { 2 - 4 } & $1,000-1,200$ & $4.7(2.3-9.3)$ & 2 \\
\cline { 2 - 4 } & $<1000$ & $6.9(3.3-14.5)$ & 3 \\
\hline \multirow{3}{*}{ Gestational age (wk) } & $>27$ & 1 (ref. cat.) & 0 \\
\cline { 2 - 4 } & $\leq 27$ & $3.7(1.9-7.1)$ & 2 \\
\hline \multirow{3}{*}{ Gender } & Female & 1 (ref. cat.) & 0 \\
\cline { 2 - 4 } & Male & $2.0(1.1-3.5)$ & 1 \\
\hline \multirow{3}{*}{ RsPDA } & Absent & 1 (ref. cat.) & 0 \\
\cline { 2 - 4 } & Present & $9.7(5.1-18.5)$ & 3 \\
\hline \multirow{3}{*}{ Hypotension } & Absent & 1 (ref. cat.) & 0 \\
\cline { 2 - 4 } & Present & $3.2(1.8-5.8)$ & 1 \\
\hline IVH/GMH & Absent & 1 (ref. cat.) & 0 \\
\cline { 2 - 4 } & Present & $5.6(2.4-13.2)$ & 2 \\
\hline & Absent & 1 (ref. cat.) & 0 \\
\cline { 2 - 4 } & Present & $3.0(1.4-6.7)$ & 1 \\
\hline
\end{tabular}

Abbreviations: BPD, bronchopulmonary dysplasia; $\mathrm{Cl}$, confidence interval; HsPDA, hemodynamically significant PDA; IVH/GMH, intraventricular/ germinal matrix hemorrhage; RDS, respiratory distress syndrome.

${ }^{a}$ Risk of BPD relative to the reference category (ref. cat.) with $95 \%$ confidence intervals $(95 \% \mathrm{Cl})$. 
Table 3 BPD-TM score

\begin{tabular}{|l|l|l|l|l|}
\hline Score & $\mathbf{0}$ & $\mathbf{1}$ & $\mathbf{2}$ & $\mathbf{3}$ \\
\hline Birth weight $(\mathrm{g})$ & $\geq 1,200$ & & $<1,200$ & $<1,000$ \\
\hline Gestational age (wk) & $>27$ & & $\leq 27$ & \\
\hline Gender & Female & Male & & Present \\
\hline HsPDA & Absent & & & \\
\hline RDS & Absent & Present & & \\
\hline Hypotension & Absent & & Present & \\
\hline IVH/GMH & Absent & Present & & \\
\hline
\end{tabular}

Abbreviations: BPD, bronchopulmonary dysplasia; HsPDA, hemodynamically significant patent ductus arteriosus; IVH/GMH, intraventricular/germinal matrix hemorrhage; RDS, respiratory distress syndrome.

Table 4 Comparison of the groups according to the scoring parameters

\begin{tabular}{|l|l|l|l|}
\hline & $\begin{array}{l}\text { Derivation cohort } \\
n=652\end{array}$ & $\begin{array}{l}\text { Validation cohort } \\
n=172\end{array}$ & $p$-Value \\
\hline Birth weight (g) (range) & $1,218 \pm 220(585-1,500)$ & $1,102 \pm 251(470-1,500)$ & $<0.001$ \\
\hline Gestational age (wk) (range) & $29.4 \pm 1.9(24.3-32)$ & $28.9 \pm 2.3(24.1-32)$ & 0.012 \\
\hline Gestational age $<27$ wks, $n$ (\%) & $58(8.9)$ & $42(24.4)$ & $<0.001$ \\
\hline Gender (male), $n$ (\%) & $318(48.8)$ & $89(51.7)$ & 0.49 \\
\hline RDS, $n$ (\%) & $189(29)$ & $106(61.6)$ & $<0.001$ \\
\hline HsPDA, $n$ (\%) & $115(17.6)$ & $41(23.8)$ & 0.07 \\
\hline Hypotension, $n$ (\%) & $62(9.5)$ & $27(15.7)$ & 0.02 \\
\hline IVH/GMH (all grades), $n$ (\%) & $69(10.6)$ & $27(15.7)$ & 0.06 \\
\hline BPD, $n$ (\%) & \multicolumn{2}{l|}{} \\
\hline Any & $150(23.0)$ & $54(31.4)$ & 0.023 \\
\hline Mild & $99(15.2)$ & $37(21.5)$ & 0.047 \\
\hline Moderate & $32(4.9)$ & $13(7.6)$ & 0.17 \\
\hline Severe & $19(2.9)$ & $4(2.3)$ & 0.68 \\
\hline
\end{tabular}

Abbreviations: BPD, bronchopulmonary dysplasia; HsPDA, hemodynamically significant patent ductus arteriosus; IVH/GMH, intraventricular/germinal matrix hemorrhage; RDS, respiratory distress syndrome.

may facilitate his/her communication with the parents. For a clinician, it is important to predict this condition to take preventive measures or to start the therapy early in the course of the disease before any irreversible change occurs. For a researcher, it is important to stratify at risk populations of neonates for clinical trials appropriately and to optimize study design by discriminatory selection of higher risk from lower risk infants. A desirable neonatal scoring system should be used easily, applied early in the course of hospitalization, be reproducible and it should be useful for all neonates. ${ }^{22,23}$ An ideal method to predict BPD would have high positive and negative predictive values and be applicable very early in life. ${ }^{5}$ BPD-TM score carries most of these features. The ability of a score to discriminate is very important and it is measured by the area under the ROC curve. ${ }^{24} \mathrm{~A}$ value above 0.8 indicates that the score may be useful in practice. In the BPD-TM score, area under the curve (AUC) values for the derivation and the validation cohorts were 0.930 and 0.903 satisfying these criteria.
Reproducibility of the scoring system is very important. We have validated our score at a different time period in the same unit. Although the clinical features of the infants differed between these time periods, BPD-TM score had similar ROC curves and predictive capacity in both populations. Nonetheless, this scoring system needs to be validated externally in a different unit, as well, since it was a limitation of our study failing to find such a comparison group.

Another limitation of this study is the low number of infants with gestational age less than 27 weeks (8.9\%) especially in the derivation cohort. This was probably the reason why the BPD incidence was low (23\%) in the derivation cohort. However, in the validation cohort nearly a quarter of the infants were less than 27 weeks and the incidence of BPD was $31.4 \%$, where the scoring was also shown to work to adequately predict the risk.

In fact, irreversible damage to the lungs starts even during in utero period. The interventions to prevent these changes should start in the delivery room routinely such as early CPAP 


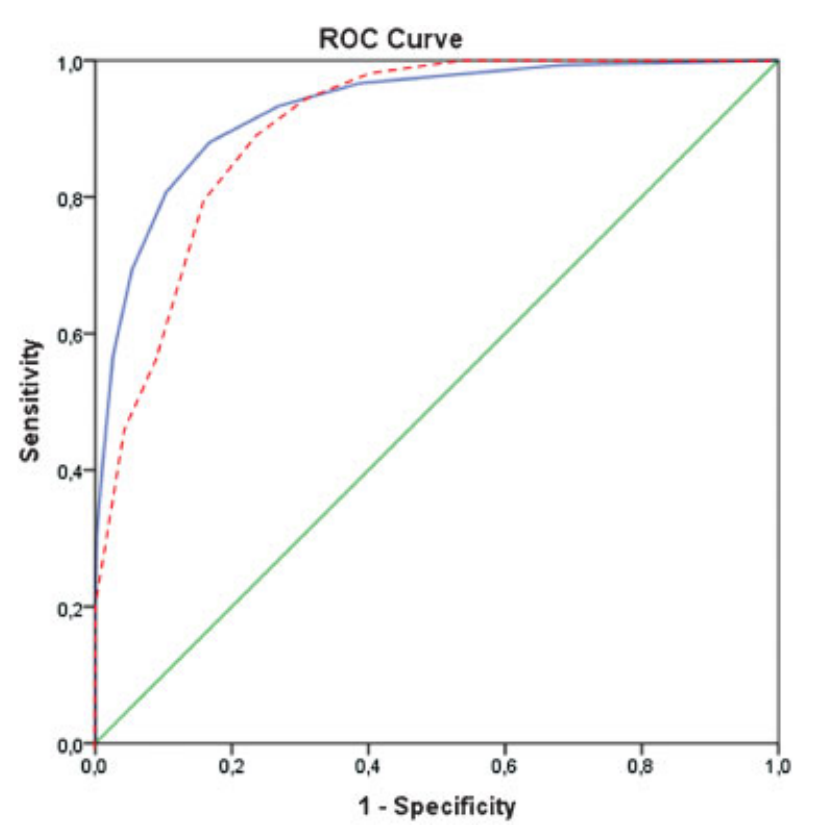

Fig. 2 ROC curves for BPD-TM score for the derivation (solid) and validation (dashed) datasets in predicting BPD. BPD, bronchopulmonary dysplasia; ROC, receiver operator characteristics.

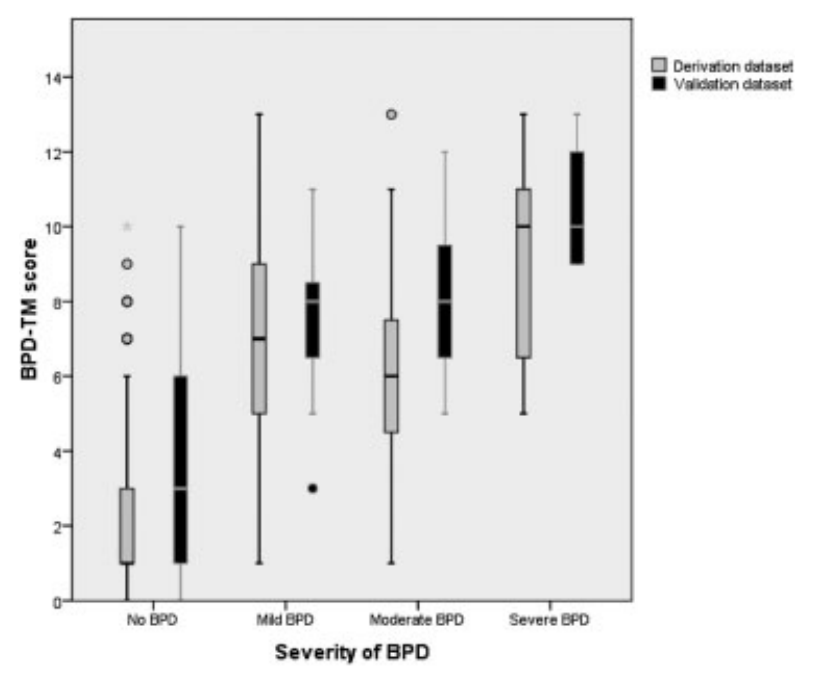

Fig. 3 The BPD-TM scores according to the severity of BPD both in the derivation and validation dataset. (The horizontal line shows median values, while the error bars indicate the lowest and highest nonoutlier observations.) BPD, bronchopulmonary dysplasia.

and noninvasive ventilation. However, benefits of postnatal treatment strategies might depend on the baseline BPD risk, such as postnatal steroids, used for the prevention of BPD after the 1st postnatal week, which outweighs the risks in only populations at high risk for BPD. A meta-analysis of randomized controlled trials of postnatal steroid therapy for prevention of BPD in preterm infants that reported rates of death or cerebral palsy (CP) demonstrated that with risks for BPD below 35\%, corticosteroid treatment significantly increased the risk of death or $\mathrm{CP}$, whereas with risks for BPD exceeding $65 \%$, it reduced this risk. ${ }^{25}$ Therefore, it may be reasonable to use postnatal steroids in preterm infants with
BPD-TM score of more than 6 (high intermediate and high risk infants).

Different scoring systems have been developed to predict BPD. ${ }^{2-11,26-28}$ Roentgenographic scores were found to be significantly correlated with clinical scores at 21 days of age $^{28}$ which is very late to perform a score for prediction of BPD. Yuksel et al used chest X-ray at 7 days of life and reached only $71 \%$ sensitivity and $88 \%$ specificity. ${ }^{11}$ On the other hand, Noack et al who combined radiological and cytological findings failed to achieve $70 \%$ for both. ${ }^{10}$ Besides, interpreting radiographic findings makes the model rather subjective and in some systems had no discriminatory value. ${ }^{4}$

Using respiratory parameters such as peak inspiratory pressure (PIP) and inspired oxygen concentration (Fio2) may be difficult to generalize as target saturation and blood gas values may change from one unit to the other. That is, these scoring systems are not independent of variations in clinical practice. Romagnoli et $\mathrm{al}^{6}$ used birth weight, PIP, and Fio2 on the 3rd and 5th postnatal day and obtained a high AUC 0.96 with a predictive value of $94 \%$. Their scoring system also correlated with the severity of lung disease just like our BPDTM score. However, they did not mention their saturation target in that study.

In the study of Sinkin et al 7 to $9 \%$ of the infants in the minimal risk category and 60 to $85 \%$ of the infants in high risk category developed BPD. ${ }^{12}$ The negative and positive predictive values of the BPD-TM score were above $90 \%$ and they are highly satisfactory.

Another scoring system based on respiratory support requirements was calculated by multiplying the Fio 2 by the level of respiratory support. ${ }^{26}$ The areas under the ROC curves reached 0.97 only on the 7th day like the ones in Ryan et al study. ${ }^{4}$

The largest multicenter study on the prediction of BPD used gestational age, birth weight, race and ethnicity, sex, respiratory support, and Fio2. ${ }^{29}$ They developed a computerbased prediction tool model starting from the 1st day of life where you have to enter these parameters as inputs and obtain the estimated probabilities of BPD or death. Prediction improved with advancing postnatal age, increasing from a $\mathrm{C}$ statistic of 0.793 on day 1 to a maximum of 0.854 on day 28 . However, our scoring system may be more practical to use in parts of the world where portable computing systems are not available at the bed side, or where race and ethnicity is not perceived and recorded the same way as in North America.

The parameters used in BPD-TM scoring system are independent of variations in clinical practice. Their definitions do not change from one unit to another and it is a very easily applicable system based on objective data. It is also correlated with the severity of BPD which is also important in clinical practice. Apart from these, this study is the highest case series of a single center from which a scoring system to predict BPD was derived.

It is important to remember that even the best scoring systems are not completely accurate. No mathematical formula can totally capture the complex clinical processes. ${ }^{21}$ Nevertheless, our score worked very well in our population. With this easy to use and reliable scoring system, one can predict the neonate at risk for BPD at 72 hours of life and 
direct preventive measures toward these infants earlier in the course of the disease. However, it would be wise to test the functionality of the BPD-TM score in other neonatology units to confirm its reliability on different populations. Future studies can be directed at the long-term follow-up of highrisk infants to examine the pulmonary function of these infants.

\section{References}

1 Stoll BJ, Hansen NI, Bell EF, et al; Eunice Kennedy Shriver National Institute of Child Health and Human Development Neonatal Research Network. Neonatal outcomes of extremely preterm infants from the NICHD Neonatal Research Network. Pediatrics 2010;126(3):443-456

2 Farstad T, Bratlid D. Incidence and prediction of bronchopulmonary dysplasia in a cohort of premature infants. Acta Paediatr 1994;83(1):19-24

3 Kim YD, Kim EA, Kim KS, Pi SY, Kang W. Scoring method for early prediction of neonatal chronic lung disease using modified respiratory parameters. J Korean Med Sci 2005;20(3):397-401

4 Ryan SW, Wild NJ, Arthur RJ, Shaw BNJ. Prediction of chronic neonatal lung disease in very low birthweight neonates using clinical and radiological variables. Arch Dis Child Fetal Neonatal Ed 1994;71(1):F36-F39

5 Yoder BA, Anwar MU, Clark RH. Early prediction of neonatal chronic lung disease: a comparison of three scoring methods. Pediatr Pulmonol 1999;27(6):388-394

6 Romagnoli C, Zecca E, Tortorolo L, Vento G, Tortorolo G. A scoring system to predict the evolution of respiratory distress syndrome into chronic lung disease in preterm infants. Intensive Care Med 1998;24(5):476-480

7 Subhedar NV, Hamdan AH, Ryan SW, Shaw NJ. Pulmonary artery pressure: early predictor of chronic lung disease in preterm infants. Arch Dis Child Fetal Neonatal Ed 1998;78(1):F20-F24

8 Bhutani VK, Abbasi S. Relative likelihood of bronchopulmonary dysplasia based on pulmonary mechanics measured in preterm neonates during the first week of life. J Pediatr 1992;120(4 Pt 1): 605-613

9 Coates AL, Vallinis P, Mullahoo K, Seddon P, Davis GM. Pulmonary impedance as an index of severity and mechanisms of neonatal lung disease. Pediatr Pulmonol 1994;17(1):41-49

10 Noack G, Mortensson W, Robertson B, Nilsson R. Correlations between radiological and cytological findings in early development of bronchopulmonary dysplasia. Eur J Pediatr 1993;152(12): 1024-1029

11 Yuksel B, Greenough A, Karani J. Prediction of chronic lung disease from the chest radiograph appearance at seven days of age. Acta Paediatr 1993;82(11):944-947

12 Sinkin RA, Cox C, Phelps DL. Predicting risk for bronchopulmonary dysplasia: selection criteria for clinical trials. Pediatrics 1990; 86(5):728-736
13 Newton ER. Chorioamnionitis and intraamniotic infection. Clin Obstet Gynecol 1993;36(4):795-808

14 Ovali F. Intrauterine growth curves for Turkish infants born between 25 and 42 weeks of gestation. J Trop Pediatr 2003; 49(6):381-383

15 Shankaran S. Hypoxic-ischemic encephalopathy and novel strategies for neuroprotection. Clin Perinatol 2012;39(4):919-929

16 Manning FA. Fetal biophysical profile. Obstet Gynecol Clin North Am 1999;26(4):557-577

17 Hamvas A. Pathophysiology and management of respiratory distress syndrome. In: Martin RJ, Fanaroff AA, Walsh MC, eds. Fanaroff and Martin's Neonatal-Perinatal Medicine Diseases of the Fetus and Infant. St. Louis: Elsevier-Mosby; 2011: 1106-1116

18 Dempsey EM, Al Hazzani F, Barrington KJ. Permissive hypotension in the extremely low birthweight infant with signs of good perfusion. Arch Dis Child Fetal Neonatal Ed 2009;94(4): F241-F244

19 Papile LA, Burstein J, Burstein R, Koffler H. Incidence and evolution of subependymal and intraventricular hemorrhage: a study of infants with birth weights less than 1,500 gm. J Pediatr 1978; 92(4):529-534

20 Evans N. Diagnosis of the preterm patent ductus arteriosus: clinical signs, biomarkers, or ultrasound? Semin Perinatol 2012; 36(2):114-122

21 Jobe AH, Bancalari E. Bronchopulmonary dysplasia. Am J Respir Crit Care Med 2001;163(7):1723-1729

22 Dorling JS, Field DJ, Manktelow B. Neonatal disease severity scoring systems. Arch Dis Child Fetal Neonatal Ed 2005;90(1): F11-F16

23 Fleisher BE, Murthy L, Lee S, Constantinou JC, Benitz WE, Stevenson DK. Neonatal severity of illness scoring systems: a comparison. Clin Pediatr (Phila) 1997;36(4):223-227

24 van Erkel AR, Pattynama PM. Receiver operating characteristic (ROC) analysis: basic principles and applications in radiology. Eur J Radiol 1998;27(2):88-94

25 Doyle LW, Halliday HL, Ehrenkranz RA, Davis PG, Sinclair JC. Impact of postnatal systemic corticosteroids on mortality and cerebral palsy in preterm infants: effect modification by risk for chronic lung disease. Pediatrics 2005;115(3):655-661

26 May C, Kavvadia V, Dimitriou G, Greenough A. A scoring system to predict chronic oxygen dependency. Eur J Pediatr 2007;166(3): 235-240

27 Madan A, Brozanski BS, Cole CH, Oden NL, Cohen G, Phelps DL. A pulmonary score for assessing the severity of neonatal chronic lung disease. Pediatrics 2005;115(4):e450-e457

28 Toce SS, Farrell PM, Leavitt LA, Samuels DP, Edwards DK. Clinical and roentgenographic scoring systems for assessing bronchopulmonary dysplasia. Am J Dis Child 1984;138(6):581-585

29 Laughon MM, Langer JC, Bose CL, et al; Eunice Kennedy Shriver National Institute of Child Health and Human Development Neonatal Research Network. Prediction of bronchopulmonary dysplasia by postnatal age in extremely premature infants. Am J Respir Crit Care Med 2011;183(12):1715-1722 